\title{
Effect of Exercise on Kidney Function, Oxidative Stress, and Inflammation in Type 2 Diabetic KK- $A^{y}$ Mice
}

\author{
Yuji Ishikawa, Tomohito Gohda, Mitsuo Tanimoto, Keisuke Omote, \\ Masako Furukawa, Saori Yamaguchi, Maki Murakoshi, Shinji Hagiwara, \\ Satoshi Horikoshi, Kazuhiko Funabiki, and Yasuhiko Tomino
}

\begin{abstract}
Division of Nephrology, Department of Internal Medicine, Juntendo University Faculty of Medicine, 2-1-1 Hongo, Bunkyo-ku, Tokyo 113-8421, Japan
\end{abstract}

Correspondence should be addressed to Yasuhiko Tomino, yasu@juntendo.ac.jp

Received 28 March 2012; Revised 17 May 2012; Accepted 4 June 2012

Academic Editor: Mark Emmanuel Cooper

Copyright (C) 2012 Yuji Ishikawa et al. This is an open access article distributed under the Creative Commons Attribution License, which permits unrestricted use, distribution, and reproduction in any medium, provided the original work is properly cited.

\begin{abstract}
Exercise is recommended for the management of type 2 diabetes, but its effects on diabetic nephropathy (DN) are still unknown. We hypothesized that appropriate exercise improves early DN via attenuation of inflammation and oxidative damage. Type 2 diabetic KK- $A^{y}$ mice, a spontaneous DN model, underwent two different kinds of exercise (i.e., moderate and low intensity). Sedentary mice or those undergoing an exercise regimen causing no significant body weight loss were used. We examined the urinary excretion of albumin, number of podocytes and macrophages, renal expressions of HIF- $1 \alpha$ and MCP-1, and biomarkers of oxidative stress such as urinary $8-\mathrm{OHdG}$ and serum SOD. Exercise reduced urinary levels of albumin and also maintained the number of podocytes in the exercised KK- $A^{y}$ mice independently of improvements of overweight and hyperglycemia, although moderate-intensity exercise increased expression of HIF- $1 \alpha$. Sedentary KK- $A^{y}$ mice showed increased expression of MCP-1 and infiltration of macrophage, increased urinary 8-OhdG, and decreased serum SOD levels compared with exercised KK- $A^{y}$ mice. On the whole, low-intensity exercise attenuates progression of early DN without affecting marked renal ischemia. Reduction rates of urinary albumin and maintained podocyte numbers, with parallel improvements in oxidative damage and inflammation, are related to beneficial effects of exercise in diabetic kidney disease.
\end{abstract}

\section{Introduction}

Recent studies suggest that a chronic inflammatory process and oxidative stress promote the progression of diabetic nephropathy (DN) [1-4]. We have also showed the presence of macrophage infiltration and increased MCP-1 expressions and levels in glomeruli and urine of $\mathrm{KK}-A^{y}$ mice, a frequently used animal model of type 2 diabetes (T2D) $[5,6]$. Furthermore, urinary 8-OHdG, a marker of oxidative DNA damage, was also increased in this mouse model [7].

Lifestyle modification, especially appropriate exercise, is recommended for the management of T2D through improvements of metabolic risk factors such as blood pressure, blood glucose, plasma lipids, and oxidative stress markers. On the other hand, this also consumes considerable amounts of oxygen, leading to production of high levels of reactive oxygen species (ROS). There is also evidence that
ROS and high glucose exposure contribute to podocyte apoptosis in experimental DN [8]. It is considered that exercise-induced proteinuria is usually not permanent but evanescent $[9,10]$. Moreover it is little known that moderate exercise has adverse effect on the renal function [11-14]. Several studies reported that exercise showed renoprotective effects in both types of diabetic subjects as well as in animal models of DN, although detailed mechanism of action by which exercise has a favorable influence on renal function is not yet fully understood [15-18].

In 1969 , the KK- $A^{y}$ mouse was established by Nishimura [19]. This mouse was produced by the transfer of the yellow obese gene $\left(A^{y}\right.$ allele) into the KK mouse. Because the diabetic feature in the KK- $A^{y}$ mouse is more severe than that in the KK mouse, this mouse is widely used as an experimental model for T2D. KK- $A^{y}$ mice spontaneously exhibit T2D associated with hyperglycemia, glucose intolerance, 
hyperinsulinemia, obesity, and microalbuminuria. Renal lesions in $\mathrm{KK}-A^{y}$ mice closely resemble those in human DN with glomeruli exhibiting diffuse mesangial hyperplasia with mesangial cell proliferation, segmental sclerosis, and overexpression of TGF $\beta-1$ at 20 weeks of age. We have reported that the KK- $A^{y}$ mouse is a suitable model for the study of DN in humans $[6,7,20-22]$.

In the present study, we hypothesized that appropriate exercise may improve early DN via attenuation of the expression of inflammation and oxidative stress in the kidneys of $\mathrm{KK}-A^{y}$ mice.

\section{Materials and Methods}

2.1. Experimental Animals and Protocols. Eight-week-old male diabetic KK- $A^{y} / \mathrm{Ta}$ Jcl mice were purchased from CLEA Japan (Tokyo, Japan). We also purchased the same age male $\mathrm{KK} / \mathrm{Ta} \mathrm{Jcl}$ mice as a control for the KK- $A^{y}$ mouse. The mice were individually housed in plastic cages with free access to food (rodent pellet diet NMF; $348 \mathrm{kcal} / 100 \mathrm{~g}$, containing $5.5 \%$ crude fat) and water throughout the experimental periods. All mice were maintained in the same room under conventional conditions with a regular 12-hour light/dark cycle and temperature controlled at $24 \pm 1^{\circ} \mathrm{C}$. All experiments were performed according to the guidelines of the Animal Care Committee of Juntendo University.

After acclimating to new surroundings and running on a treadmill (Osaka Micro System, Osaka, Japan) for 4 weeks, KK- $A^{y}$ mice were divided into three groups: (1) sedentary group (no exercise), (2) low-intensity exercise group, and (3) moderate-intensity exercise group ( $n=10-13$ per group). Mice were exercised at $10 \mathrm{~m} / \mathrm{min}$ for an hour 5 days a week in the moderate-intensity group or $5 \mathrm{~m} / \mathrm{min}$ for 30 minutes 3 days a week in the low-intensity group for 8 weeks. Nonexercised $\mathrm{KK}-A^{y}$ and $\mathrm{KK}$ mice were placed on nonrotating wheels for the same duration. The experimental protocol was terminated when the mice reached 20 weeks of age.

2.2. Biochemical Measurements. The urinary albumin-creatinine ratio (ACR), body weight (BW), and hemoglobin Alc (HbAlc) were measured at 12 and 20 weeks of age. The levels of urinary 8-hydroxydeoxyguanosine (8-OHdG), $\mathrm{N}$-acetyl$\beta$-D-glucosaminidase (NAG), serum creatinine, superoxide dismutase (SOD), and creatinine clearance (Ccr) were measured at 20 weeks of age. Urinary samples were collected for 24 hours using a metabolic cage (mouse metabolic cage, CLEA Japan). Urinary albumin and creatinine samples were measured by immunoassay (DCA 2000 System; Bayer Diagnostics, Elkhart, IN). Glucose levels in blood obtained from the retroorbital sinus were measured using Glucocard (Kyoto Daiichi Kagaku, Kyoto, Japan). HbAlc was also measured by immunoassay (DCA 2000 system, Bayer Diagnostics). Serum creatinine was measured by an autoanalyzer (Fuji Dry-chem 5500; Fujifilm, Tokyo, Japan). Urinary 8-OHdG level was measured by an enzyme-linked immunosorbent assay (Fushimi Pharmaceutical, Kagawa, Japan) [23]. Serum SOD activity (SOD Assay Kit-WST, Dojindo Molecular Technologies, Tokyo, Japan) was measured by enzyme immunoassay. Ccr was estimated as the ratio of daily urinary creatinine excretion to plasma creatinine concentration and then expressed as milliliters per minute per square meter of surface area.

2.3. Immunohistochemical Staining for Hypoxia Inducible Factor (HIF)-1 $\alpha$, Monocyte Chemotactic Protein (MCP)-1, CD68, and CD204. The mice were sacrificed at 20 weeks of age. The kidneys were retrogradely perfused with saline via the abdominal aorta for 5 minutes at a pressure of about $150 \mathrm{mmHg}$ without prior flushing of the vasculature. Immunostaining of the frozen sections or paraffin-embedded sections was performed. Renal tissues were snap frozen in an optimal cutting temperature compound and cut into 3$\mu \mathrm{m}$-thick sections. Formalin-fixed and paraffin-embedded tissues were cut at $2 \mu \mathrm{m}$. Immunohistochemical studies were performed using commercially available antibodies as follows: mouse monoclonal anti-HIF- $1 \alpha$ antibody (ab-1; Abcam, MA, USA), goat polyclonal anti-MCP-1 antibody (sc-1784; Santa Cruz, CA, USA), rat monoclonal anti-CD68 (the marker for pan macrophage) (MCA1957; AbD Serotec, Oxford, UK), and rat monoclonal anti-CD204 (the marker for M2 macrophage) (MCA1322; AbD Serotec, Oxford, UK). HIF- $1 \alpha$ stained sections were then blocked with mouse Ig blocking reagent (MKB-2213; Vector Laboratories, CA, USA). The other stained sections were blocked by blocking solution ( $2 \%$ fetal bovine serum and $10 \%$ normal goat serum in PBS). The sections were incubated with secondary antibody: anti-goat $\operatorname{IgG}$, anti-mouse $\operatorname{IgG}$, and anti-rat $\operatorname{IgG}$, respectively (Nichirei, Tokyo, Japan). Secondary antibody was visualized by light microscopy with diaminobenzidine.

The HIF- $1 \alpha$ staining of at least 20 randomly selected fields $(\times 200)$ from each mouse was quantified using the KS400 version 4.0 image analysis system (KS-400; Carl Zeiss Vision, Munich, Germany). The numbers of M1, M2, and MCP-1-positive cells were counted in at least 20 randomly selected fields $(\times 200)$ and/or at least 20 glomeruli. Analyses were performed by two investigators in a blinded fashion $[6,24]$.

2.4. Quantitative Analysis of MCP-1 $m R N A$ by Real-Time PCR. Real-time PCR was also used to evaluate MCP-1 mRNA expression in whole kidneys of mice at 20 weeks of age. Total RNA was extracted from whole kidneys using the RNeasy Mini Kit (Qiagen K.K., Tokyo, Japan). Complementary DNA was synthesized using random hexamers (Quantum RNA kit; Ambion, Austin, Tex., USA). The complementary DNA was further amplified by a real-time polymerase chain reaction (PCR) system (ABI Prism 7500 Real-Time PCR System; PerkinElmer, Foster City, Calif., USA). Initial template concentration was derived from the cycle number at which the fluorescent signal crosses a threshold in the exponential phase of the PCR reaction. Relative gene expression was determined based on the threshold cycles (Ct values). The PCR parameters were $95^{\circ} \mathrm{C}$ for $10 \mathrm{~min}, 50$ cycles at $95^{\circ} \mathrm{C}$ for $15 \mathrm{~s}$, and $60^{\circ} \mathrm{C}$ for $60 \mathrm{~s}$. Primers and fluorogenic probes of MCP-1 were obtained from TaqMan Gene Expression Assays, Applied Biosystems (Foster City, Calif., USA). The assay identity 
TABLE 1: Biochemical parameters in each mouse at 12 and 20 weeks of age.

\begin{tabular}{|c|c|c|c|c|}
\hline & KK & $\mathrm{KK}-A^{y}$ & $\mathrm{KK}-A^{y}$ & $\mathrm{KK}-A^{y}$ \\
\hline Number & 9 & 11 & 10 & 13 \\
\hline Intensity of exercise & Sedentary & Sedentary & Low & Moderate \\
\hline \multicolumn{5}{|l|}{12 weeks of age } \\
\hline Body weight (g) & $31.9 \pm 0.6^{\ddagger}$ & $39.9 \pm 2.4$ & $40.9 \pm 2.2$ & $40.3 \pm 2.1$ \\
\hline HbA1c (\%) & $4.0 \pm 0.1^{\ddagger}$ & $7.0 \pm 0.9$ & $7.4 \pm 1.1$ & $6.7 \pm 1.4$ \\
\hline Urinary albumin $(\mathrm{mg} / \mathrm{g} \cdot \mathrm{Cr})$ & $86 \pm 120^{\ddagger}$ & $398 \pm 203$ & $434 \pm 177$ & $402 \pm 160$ \\
\hline \multicolumn{5}{|l|}{20 weeks of age } \\
\hline Body weight (g) & $36.7 \pm 1.2^{\ddagger}$ & $44.4 \pm 3.6$ & $46.3 \pm 2.9$ & $44.5 \pm 3.3$ \\
\hline FBG (mg/dL) & $97 \pm 15$ & $87 \pm 29$ & $72 \pm 19$ & $91 \pm 25$ \\
\hline HbAlc $(\%)$ & $4.4 \pm 0.4^{\ddagger}$ & $8.8 \pm 1.1$ & $9.5 \pm 1.3$ & $8.4 \pm 1.3$ \\
\hline Serum creatinine (mg/dL) & $0.14 \pm 0.06$ & $0.16 \pm 0.05$ & $0.15 \pm 0.05$ & $0.16 \pm 0.05$ \\
\hline Urinary albumin $(\mathrm{mg} / \mathrm{g} \cdot \mathrm{Cr})$ & $98 \pm 43^{\ddagger}$ & $1104 \pm 569$ & $726 \pm 388$ & $833 \pm 370$ \\
\hline Urinary NAG $(\mathrm{U} / \mathrm{mg} / \mathrm{L} \cdot \mathrm{Cre})$ & $9.7 \pm 2.0^{\ddagger}$ & $22.8 \pm 6.0$ & $16.7 \pm 4.4^{*}$ & $17.3 \pm 3.5$ \\
\hline Urinary 8-OHdG $(\mu \mathrm{g} / \mathrm{g} \cdot \mathrm{Cre})$ & $47 \pm 11^{\dagger}$ & $306 \pm 159$ & $56 \pm 42^{\dagger}$ & $97 \pm 105^{\dagger}$ \\
\hline Serum SOD $(\mathrm{U} / \mathrm{dL})$ & $243 \pm 101$ & $226 \pm 37$ & $316 \pm 40^{\dagger}$ & $326 \pm 59^{\ddagger}$ \\
\hline $\operatorname{Ccr}(\mathrm{mL} / \mathrm{min})$ & $64.7 \pm 12.3$ & $88.8 \pm 33.9$ & $82.9 \pm 29.7$ & $84.2 \pm 36.4$ \\
\hline
\end{tabular}

Data expressed as means $\pm \mathrm{SD}$. ${ }^{*} P<0.05$ versus KK- $A^{y}$ sedentary, ${ }^{\dagger} P<0.01$ versus KK- $A^{y}$ sedentary, ${ }^{\ddagger} P<0.001$ versus KK- $A^{y}$ sedentary.

HbAlc: hemoglobin A1c, FBG: fasting blood glucose, NAG: N-acetyl- $\beta$-D-glucosaminidase, 8-OHdG: 8-hydroxydeoxyguanosine, SOD: super oxide dismutase, and Ccr: creatinine clearance.

number of MCP-1 was Mm-00441242-m1 and GAPDH was Mn-99999915-g1.

2.5. Immunofluorescent Staining for Podocytes (WT-1). Immunofluorescent staining for podocytes in renal tissues was performed using the polyclonal rabbit anti-mouse WT-1 antibody (Santa Cruz, SC-192). Goat anti-rabbit Alexa Fluor 488 was used to visualize WT- 1 positive cells. The sections were mounted with a fluorescent mounting media (Dako Cytomation) before visualization with a Fluoview 1000 confocal microscope (Olympus, Tokyo, Japan) and FV10ASW software (version 1.3c; Olympus).

The staining of at least 20 glomeruli from each mouse was quantified using the KS-400 image analysis system. The number of podocytes per glomerular area was determined using the method of Weibel [25]. The ratio of podocyte number to glomerular area was also calculated. These examinations were performed by two investigators without knowledge of the origin of the slides, and then mean values were calculated.

2.6. Statistical Analysis. Data were expressed as mean \pm SD. Statistical differences between means were determined using Dunnett's test. A value of $P<0.05$ was considered to be statistically significant.

\section{Results}

3.1. Biochemical Parameters. Before the start of the experiment, we measured the baseline fasting blood glucose, body weight, and urinary albumin of each animal at 12 weeks of age, and confirmed that there were no significant differences in those values among sedentary and exercised $\mathrm{KK}-A^{y}$ mice. However, these parameters in sedentary KK- $A^{y}$ mice were significantly higher compared with those in sedentary KK mice (Table 1).

Biochemical parameters after the 8-week experimental protocol are also shown in Table 1. Body weight and HbAlc levels in sedentary KK- $A^{y}$ mice remained at a high level compared with those in sedentary KK mice. However, these levels did not differ among sedentary and exercised KK- $A^{y}$ mice. The levels of Ccr in KK- $A^{y}$ mice were slightly increased compared with those in KK mice. However, there were no statistically significant changes in the levels of Ccr between sedentary KK- $A^{y}$ mice and sedentary KK mice. Moreover, no significant changes were observed in the levels of Ccr between sedentary and exercised $\mathrm{KK}-A^{y}$ mice (Table 1$)$. Urinary albumin levels in the exercised $\mathrm{KK}-A^{y}$ mice tended to be lower than those in sedentary KK- $A^{y}$ mice, although the difference was not statistically significant. However, the rate of urinary albumin change from 12 to 20 weeks (Figure 1) and urinary NAG levels in KK- $A^{y}$ mice with low-intensity exercise reached statistically significant levels. Exercised KK$A^{y}$ mice had significantly lower levels of urinary 8-OHdG and higher serum SOD compared with sedentary KK- $A^{y}$ mice. These levels approached those of KK mice, although these did not differ between low- and moderate-intensity exercised KK- $A^{y}$ mice.

3.2. Immunohistochemistry of HIF- $1 \alpha, M C P-1, C D 68$, and CD204 in the Kidneys. HIF- $1 \alpha$ proteins were mainly localized in the tubules and interstitium adjacent to the medulla. Contrary to expectations, the expression of HIF- $1 \alpha$ in the moderate-intensity exercised KK- $A^{y}$ mice was significantly 


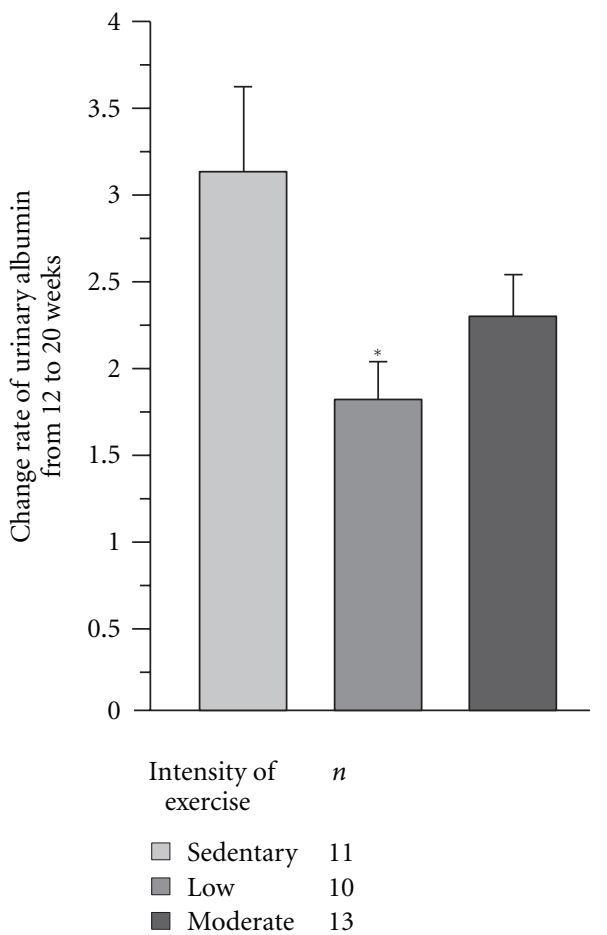

FIgURE 1: Change rate of urinary albumin from 12 to 20 weeks in $\mathrm{KK}-A^{y}$ mice. Change rate of urinary albumin at 20 weeks of age divided by that at 12 weeks of age. Low-intensity exercised KK- $A^{y}$ mice had greater change rate of urinary albumin compared with sedentary mice. ${ }^{*} P<0.05$.

higher compared with that in sedentary $\mathrm{KK}-A^{y}$ mice, although it did not differ between sedentary and lowintensity exercised KK- $A^{y}$ mice (Figure 2). Also, HIF- $1 \alpha$ expression was not observed in sedentary KK mice.

MCP-1 positive cells were localized in the proximal tubules. The numbers of MCP-1 positive cells per $1000 \mu \mathrm{m}^{2}$ in the exercised $\mathrm{KK}-A^{y}$ mice were significantly lower compared with those in the sedentary KK- $A^{y}$ mice, and came close to those of the sedentary KK mice (Figure 3 ).

The number of M2 macrophages in the renal tubulointerstitial area was not changed between sedentary and exercised KK- $A^{y}$ mice, but that of estimated M1 macrophages, calculated by subtracting the number of CD204 from CD68 positive cells in intensely exercised KK- $A^{y}$ mice, showed a significant decrease compared to the sedentary KK- $A^{y}$ mice. Although the number of CD68 and CD204 positive cells was similar in the glomeruli, the number of CD68 and CD204 positive cells in the glomeruli was much lower than that in the renal tubulointerstitial areas. This result suggests that most of macrophages are considered to be M2 macrophages in the glomeruli, and that the number of M2 macrophages showed a reduction in the low-intensity exercised KK- $A^{y}$ mice as compared to the sedentary KK- $A^{y}$ mice (Figure 4 ).

3.3. Renal Expression of MCP-1 mRNA in the Kidney. As shown in Figure 5, the level of MCP-1 mRNA 1 in the sedentary KK- $A^{y}$ mice was significantly higher than exercised KK$A^{y}$ mice and also sedentary KK mice.
3.4. Number of WT-1 Positive Cells. As shown in Figure 6, the number of glomerular WT-1 positive cells, that is podocytes, per $1000 \mu \mathrm{m}^{2}$ of glomerular areas in the sedentary KK- $A^{y}$ mice was significantly lower than that in sedentary KK mice. On the other hand, the number of podocytes in low- and moderate-intensity exercised $\mathrm{KK}-A^{y}$ mice was significantly greater than that in the sedentary KK- $A^{y}$ mice.

\section{Discussion}

In the present study, if the body weight of exercised mice did not increase compared with that of sedentary mice when mice were allowed to eat ad libitum, we defined such exercise as moderate intensity. We also demonstrated that exercise training improved urinary NAG levels as well as the change rate of urinary albumin from 12 to 20 weeks, independent of body weight and glycemic status in the kidneys of KK$A^{y}$ mice, although moderate-intensity exercise increased expression of HIF- $1 \alpha$ in the kidneys. In this study, no significant changes were observed in the levels of Ccr between sedentary KK- $A^{y}$ and exercised KK- $A^{y}$ mice. Therefore, it is indicated that the decrease of urinary albumin was not due to the reduction of renal blood flow/glomerular filtration rate, but more likely to the effect of exercise. Our running program $(10 \mathrm{~m} / \mathrm{min}$ for one hour, 5 days a week for a total of 8 weeks) was very similar but slightly less intense compared with the program of Huang et al. ( $15 \mathrm{~m} / \mathrm{min}$ for $45 \mathrm{~min}, 5$ days a week, for a total of 8 weeks) [26]. Interestingly, they also demonstrated that exercise training does not affect body weight in $\mathrm{KK}-A^{y}$ mice although the starting time of exercise differed from that in our protocol (12-20 weeks versus 8-16 weeks).

There are several mechanisms for the renoprotective effects of exercise in DN. In general, exercise training ameliorates renal function by improving metabolic factors such as plasma lipids, blood glucose, blood pressure, and body weight. It is also known to improve renal histology without altering metabolic factors in line with our present study. Boor et al. [27] demonstrated that exercise training reduced advanced glycation end products (AGEs) in both serum and kidney tissues of obese Zucker rats, an animal model of T2D and associated nephropathy, without altering inflammatory biomarkers or metabolic factors. In contrast, our study clearly showed that the exercised mice showed attenuated renal expression of MCP-1 and infiltration of macrophage in the kidneys. In general, macrophage activation is defined into two polarizations, $\mathrm{M} 1$ and $\mathrm{M} 2$, in adipose tissues [28], although the implication of these macrophages in $\mathrm{DN}$ is not yet fully understood. The M1 macrophage produces proinflammatory cytokines, such as TNF $\alpha$ and IL-6, while on the other hand, the M2 macrophage produces an antiinflammatory cytokine [29]. The number of macrophages in glomeruli and the tubulointerstitial area increased with the progression of DN. The pattern of M1 macrophage in tubulointerstitial areas was similar to that of MCP-1 positive cells, suggesting that exercise might attenuate MCP1 expression by preventing M1 macrophage infiltration, mainly in the tubulointerstitial areas. In glomeruli, contrary to our expectations, most of macrophages seemed to be 


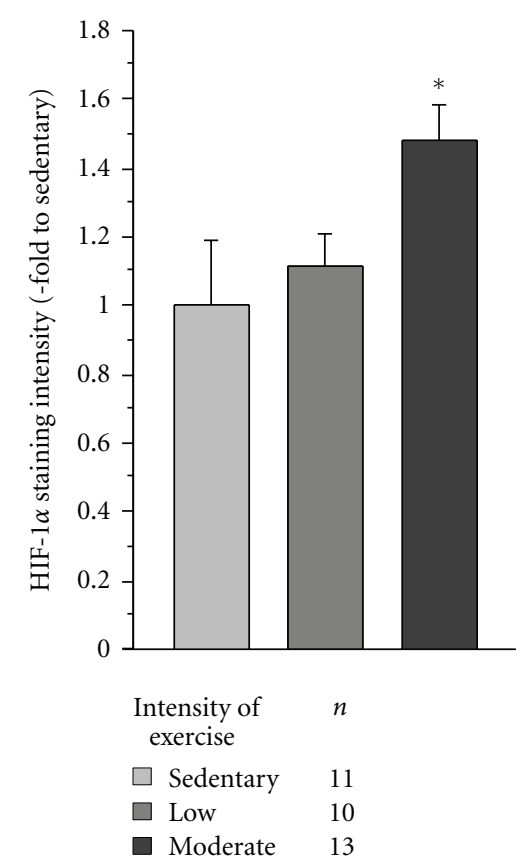

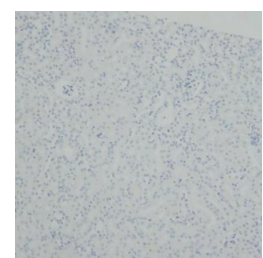

(a)

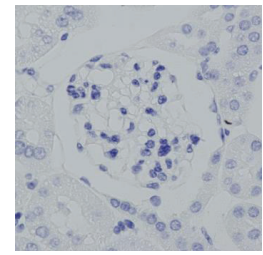

(f)

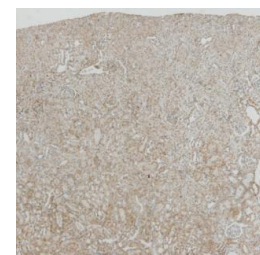

(d)

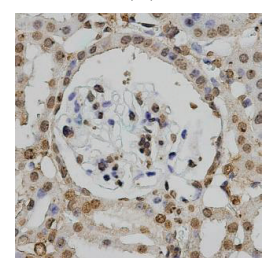

(i)

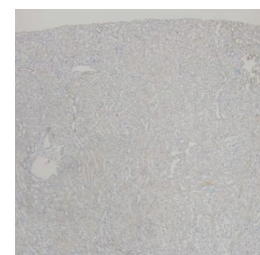

(b)

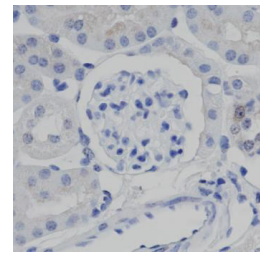

(g)

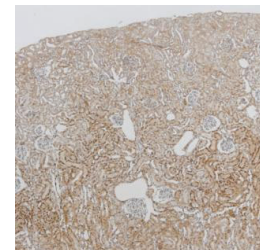

(e)

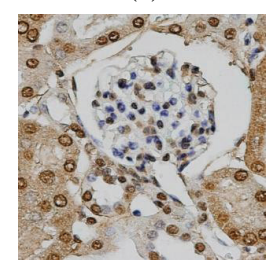

(j)

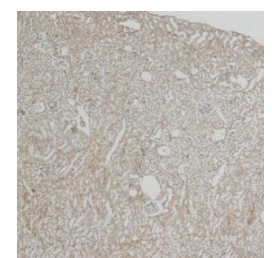

(c)

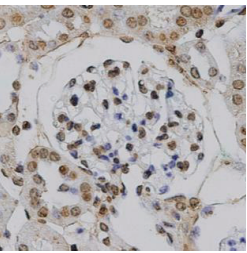

(h)

FIGURE 2: HIF- $1 \alpha$ staining intensity and staining in KK- $A^{y}$ mice with and without exercise at 20 weeks of age. HIF- $1 \alpha$ staining intensity in moderate-intensity exercised KK- $A^{y}$ mice was significantly enhanced compared with that in the sedentary KK- $A^{y}$ mice, but these levels did not differ between sedentary and low-intensity exercised KK- $A^{y}$ mice. ${ }^{*} P<0.001$. Representative HIF- $1 \alpha$ staining in the renal cortex of sedentary KK (b, g), sedentary KK- $A^{y}(\mathrm{c}, \mathrm{h})$, low-intensity exercised KK- $A^{y}(\mathrm{~d}, \mathrm{i})$, and moderate-intensity exercised KK- $A^{y}$ mice. (e, j) Staining $(\mathrm{a}, \mathrm{f})$ depicts the absence of primary antibody. Images (a-e) were taken at 40 -fold magnification and images ( $\mathrm{f}-\mathrm{j}$ ) were taken at 400-fold magnification. ${ }^{*} P<0.001$. 


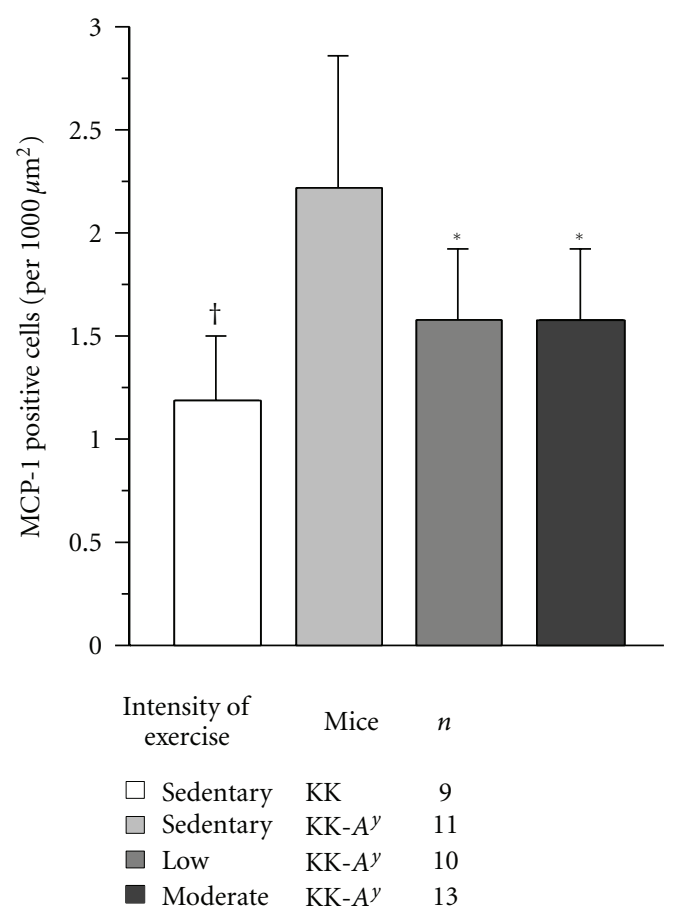

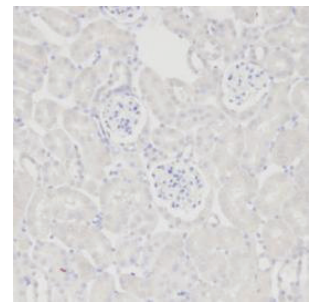

(a)

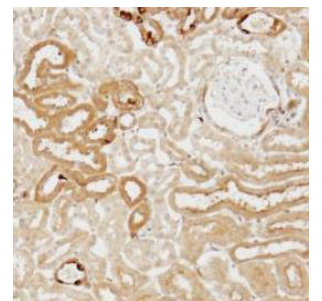

(d)

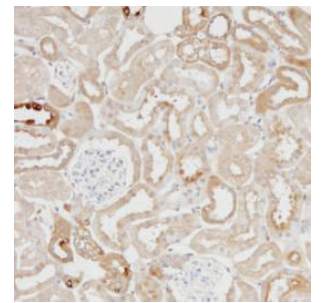

(b)

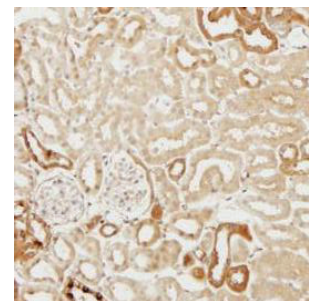

(e)

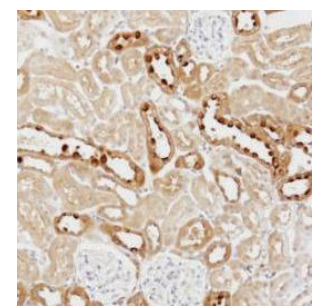

(c)

FIgURE 3: Number of MCP-1 positive cells and staining in each mouse at 20 weeks of age. The number of MCP-1 positive cells in sedentary KK- $A^{y}$ mice was significantly greater compared with that in the exercised KK- $A^{y}$ mice and also sedentary KK mice. Representative MCP-1 staining in the renal cortex of sedentary KK (b), sedentary KK- $A^{y}(\mathrm{c})$, low-intensity exercised KK- $A^{y}$ (d), and moderate-intensity exercised KK- $A^{y}$ mice. (e) Staining (a) depicts the absence of primary antibody. Images were taken at 100 -fold magnification. ${ }^{*} P<0.05^{\dagger} P<0.001$.

M2 macrophages, and these macrophages increased with the progression to $\mathrm{DN}$, although the number of pan macrophages in the glomeruli was very small compared with that in tubulointerstitial areas. It is still unknown whether the function of M2 macrophages in the kidney is different from that in adipose tissues or if M2 macrophages just increase to compensate for the increase of M1 macrophage in the kidney. Further study will be required to explain this.

It is considered that appropriate exercise increases antioxidant enzymes, although excessive exercise causes inflammation, increases oxidative stress associated with ROS, and decreases renal blood flow and glomerular filtration rate. Moien-Afshari et al. [30] demonstrated that expression of
SOD isoform depends on exercise intensity in the aorta of diabetic $\mathrm{db} / \mathrm{db}$ mice. In the present study, both intensities of exercise increased serum SOD levels, even though we did not confirm each isoform level. Moreover, both exercises decreased urinary 8-OHdG level, an oxidative stress marker. However, contrary to our expectation, low-intensity exercise was more effective than moderate-intensity exercise in terms of renal function. We require further investigation to determine appropriate exercise intensity. Although flawed, this study indicated that exercise training might attenuate podocytopenia and albuminuria, partly through antiinflammatory or/and anti-oxidant effects because it did not affect metabolic factors. 

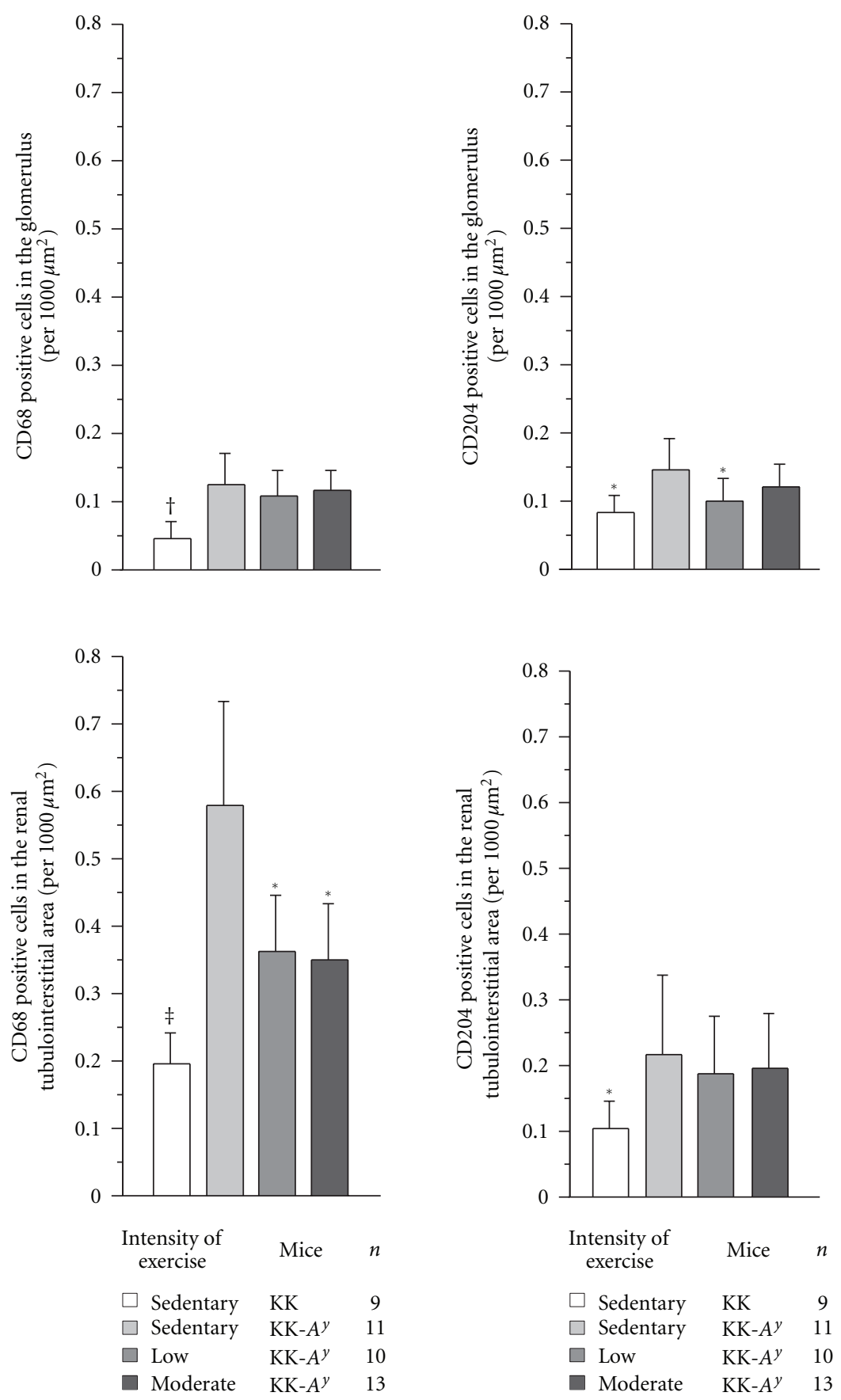

Figure 4: Number of CD68 and CD204 positive cells and staining in each mouse at 20 weeks of age. Bar charts show CD68 and CD204 positive cells in the glomeruli and tubulointerstitial area of sedentary KK (b), sedentary KK- $A^{y}$ (c), low-intensity exercised KK- $A^{y}$ (d), and moderate-intensity exercised KK- $A^{y}$ mice. (e) The number of CD68 positive cells in glomeruli of sedentary KK- $A^{y}$ mice were significantly greater compared with that of sedentary KK mice, but it did not differ between sedentary and exercised KK- $A^{y}$ mice. The number of CD68 positive cells in glomeruli of sedentary KK- $A^{y}$ mice was significantly greater compared with that of sedentary KK mice and also low-intensity exercised KK- $A^{y}$ mice, but not moderate-intensity exercised $\mathrm{KK}-A^{y}$ mice. The number of CD68 positive cells in the tubulointerstitial area of sedentary KK- $A^{y}$ mice was significantly greater compared with that of sedentary KK mice and also exercised KK- $A^{y}$ mice, but it did not differ between low- and moderate-intensity exercised KK- $A^{y}$ mice. The number of CD204 positive cells in tubulointerstitial area of sedentary KK- $A^{y}$ mice was significantly greater compared with that of sedentary KK mice, but it did not differ between sedentary and exercised KK- $A^{y}$ mice. ${ }^{*} P<0.05^{\dagger} P<0.01^{\ddagger} P<0.001$. 


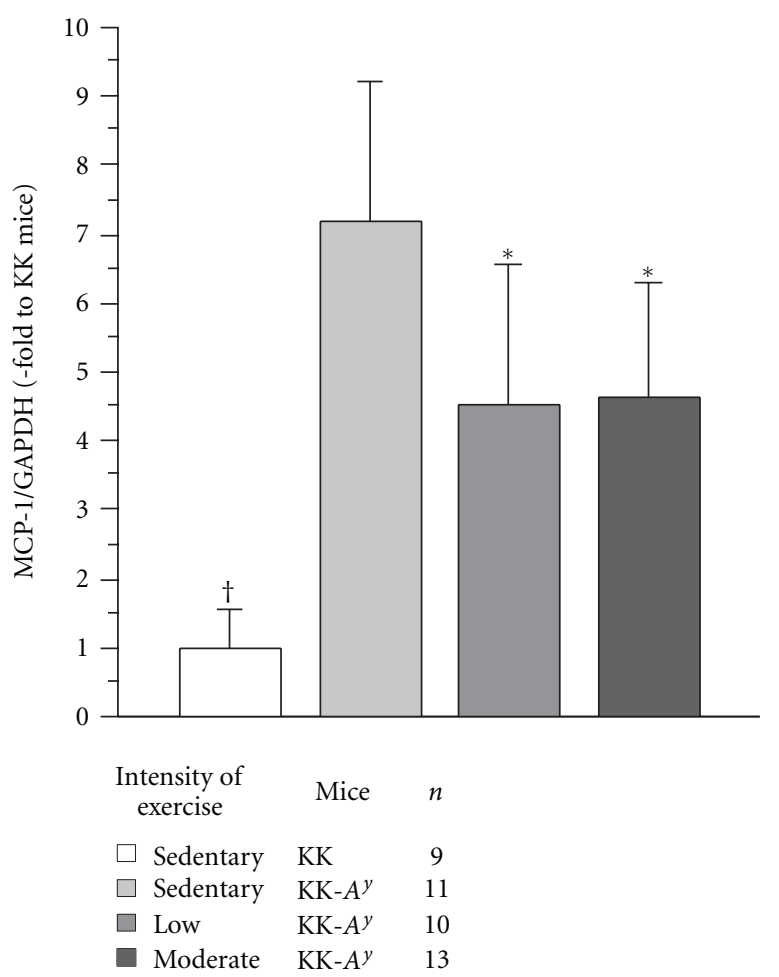

FIgURE 5: Renal expression of MCP-1 mRNA in the kidney at 20 weeks of age. The level of MCP-1 mRNA 1 in the sedentary KK- $A^{y}$ mice was significantly higher than that in exercised $\mathrm{KK}-A^{y}$ mice and also sedentary KK mice. ${ }^{*} P<0.05^{\dagger} P<0.01$.

The main limitations of our study are as follows. We did not measure blood pressure, and the beneficial effects of exercise might be partly derived from antihypertensive effects. Furthermore, we could not estimate appropriate exercise intensity in human from these results.

In conclusion, low-intensity exercise attenuates progression of early diabetic nephropathy without affecting marked renal ischemia. However, attention should be paid to renal ischemia even though albuminuria improved. Reductions in rate of urinary albumin change, urinary NAG, and maintained podocyte numbers, with parallel improvements in oxidative damage and chronic inflammation, might be related to beneficial effects of exercise in diabetic kidney disease.

\section{A list of Abbreviations}

AGEs: Advanced glycation end products

ACR: Albumin-creatinine ratio

BW: Body weight

Ccr: Creatinine clearance

DN: Diabetic nephropathy

HbA1c: Hemoglobin Alc

HIF: Hypoxia inducible factor

MCP: Monocyte chemotactic protein

NAG: $\quad \mathrm{N}$-acetyl- $\beta$-glucosaminidase

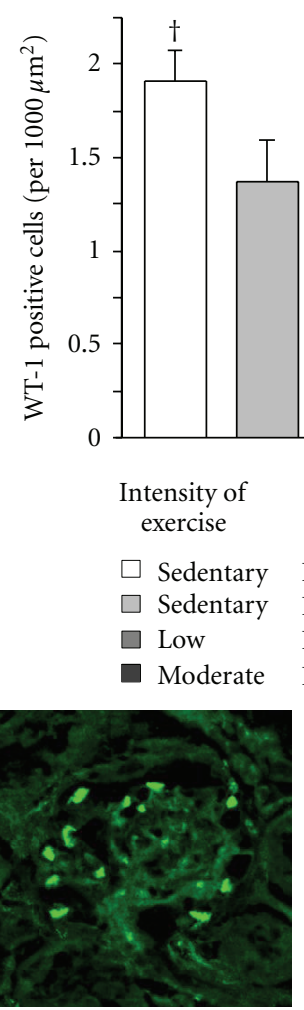

(a)

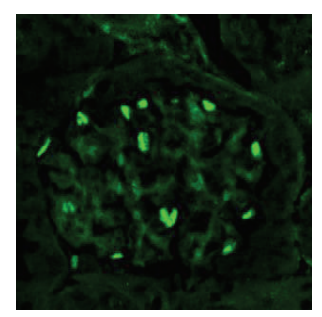

(c)

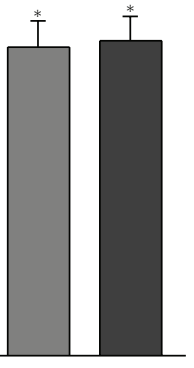

Mice $n$

KK 9

$\mathrm{KK}-A^{y} \quad 11$

$\mathrm{KK}-A^{y} \quad 10$

$\mathrm{KK}-A^{y} \quad 13$

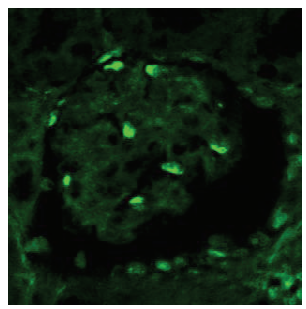

(b)

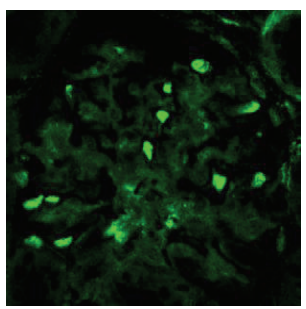

(d)
FIgURE 6: Number of WT-1 positive cells and staining in each mouse at 20 weeks of age. WT-1 positive cells in exercised KK$A^{y}$ mice were significantly maintained compared with those in the sedentary KK- $A^{y}$ mice and approached to those in KK mice. ${ }^{*} P<$ $0.01^{\dagger} P<0.001$. Representative WT-1 staining in the renal cortex of sedentary KK (a), sedentary KK- $A^{y}$ (b), low-intensity exercised KK$A^{y}$ (c), and moderate-intensity exercised KK- $A^{y}$ mice. (d) Images were taken at 400 -fold magnification.

ROS: $\quad$ Reactive oxygen species

SOD: $\quad$ Superoxide dismutase

T2D: $\quad$ Type 2 diabetes

8-OHdG: 8-hydroxydeoxyguanosine.

\section{Conflict of Interests}

The authors declare no conflict of interests.

\section{Authors' Contribution}

The design of the study was made by Y. Ishikawa, T. Gohda, M. Tanimoto, and Y. Tomino. Y. Ishikawa, K. Omote, M. 
Furukawa, and S. Yamaguchi conducted the study. Data were collected by Y. Ishikawa, K. Omote, M. Furukawa, and S. Yamaguchi. Analysis and date interpretation were conducted by Y. Ishikawa, M. Murakoshi, S. Hagiwara, and K. Funabiki. Manuscript was written by Y. Ishikawa, T. Gohda, and Y. Tomino.

\section{Acknowledgments}

The authors thank Dr. M. Takagi and Miss T. Shibata for their skillful technical support. This work was supported by Japan Society for the Promotion of Science, Grants-in-Aid for Scientific Research C (KAKENHI 21500694), and Sportology Center (a National Grant-in-Aid for the Establishment of High-Tech Research Centers in Private Universities from the Ministry of Education, Culture, Sports, Science and Technology of Japan).

\section{References}

[1] Y. Gorin, K. Block, J. Hernandez et al., "Nox4 NAD(P)H oxidase mediates hypertrophy and fibronectin expression in the diabetic kidney," Journal of Biological Chemistry, vol. 280, no. 47, pp. 39616-39626, 2005.

[2] A. A. Eid, Y. Gorin, B. M. Fagg et al., "Mechanisms of podocyte injury in diabetes role of cytochrome P450 and NADPH oxidases," Diabetes, vol. 58, no. 5, pp. 1201-1211, 2009.

[3] D. K. Singh, P. Winocour, and K. Farrington, "Oxidative stress in early diabetic nephropathy: fueling the fire," Nature Reviews Endocrinology, vol. 7, no. 3, pp. 176-184, 2011.

[4] A. Fornoni, A. Ijaz, T. Tejada, and O. Lenz, "Role of inflammation in diabetic nephropathy," Current Diabetes Reviews, vol. 4, no. 1, pp. 10-17, 2008.

[5] S. Hagiwara, Y. Makita, L. Gu et al., "Eicosapentaenoic acid ameliorates diabetic nephropathy of type 2 diabetic KKAy/Ta mice: involvement of MCP-1 suppression and decreased ERK1/2 and p38 phosphorylation," Nephrology Dialysis Transplantation, vol. 21, no. 3, pp. 605-615, 2006.

[6] I. Ohara, M. Tanimoto, T. Gohda et al., "Effect of combination therapy with angiotensin receptor blocker and 1,25dihydroxyvitamin $\mathrm{D}_{3}$ in type 2 diabetic nephropathy in KK$\mathrm{A}^{y} /$ Ta mice," Nephron. Experimental Nephrology, vol. 117, no. 4, pp. e124-e132, 2011.

[7] M. Matsumoto, M. Tanimoto, T. Gohda et al., "Effect of pitavastatin on type 2 diabetes mellitus nephropathy in KKA ${ }^{y} /$ Ta mice," Metabolism, vol. 57, no. 5, pp. 691-697, 2008.

[8] K. Susztak, A. C. Raff, M. Schiffer, and E. P. Böttinger, "Glucose-induced reactive oxygen species cause apoptosis of podocytes and podocyte depletion at the onset of diabetic nephropathy," Diabetes, vol. 55, no. 1, pp. 225-233, 2006.

[9] K. H. Koh, B. Dayanath, and J. C. Doery, "Effect of exercise on albuminuria in people with diabetes," Nephrology, vol. 16, no. 8, pp. 704-709, 2011.

[10] G. Fuiano, D. Mancuso, P. Cianfrone et al., "Can young adult patients with proteinuric IgA nephropathy perform physical exercise?" American Journal of Kidney Diseases, vol. 44, no. 2, pp. 257-263, 2004.

[11] I. Eidemak, "Exercise training and the progression of chronic renal failure," Nephron, vol. 75, no. 1, pp. 36-40, 1997.

[12] S. Ghosh, M. Khazaei, F. Moien-Afshari et al., "Moderate exercise attenuates caspase-3 activity, oxidative stress, and inhibits progression of diabetic renal disease in $\mathrm{db} / \mathrm{db}$ mice,"
American Journal of Physiology, vol. 296, no. 4, pp. F700-F708, 2009.

[13] J. M. Chiasera, K. M. Ward-Cook, S. A. McCune, and G. M. Wardlaw, "Effect of aerobic training on diabetic nephropathy in a rat model of type 2 diabetes mellitus," Annals of Clinical and Laboratory Science, vol. 30, no. 4, pp. 346-353, 2000.

[14] K. M. Ward, J. D. Mahan, and W. M. Sherman, "Aerobic training and diabetic nephropathy in the obese Zucker rat," Annals of Clinical and Laboratory Science, vol. 24, no. 3, pp. 266-277, 1994.

[15] U. Pechter, M. Ots, S. Mesikepp et al., "Beneficial effects of water-based exercise in patients with chronic kidney disease," International Journal of Rehabilitation Research, vol. 26, no. 2, pp. 153-156, 2003.

[16] C. Kornhauser, J. M. Malacara, and M. H. Macias-Cervantes, "Effect of exercise intensity on albuminuria in adolescents with Type 1 diabetes mellitus," Diabetic Medicine, vol. 29, no. 1, pp. 70-73, 2012.

[17] D. J. Leehey, I. Moinuddin, J. P. Bast et al., "Aerobic exercise in obese diabetic patients with chronic kidney disease: a randomized and controlled pilot study," Cardiovascular Diabetology, vol. 8, article 62, 2009.

[18] H. Fujita, T. Sakamoto, and K. Komatsu, "Reduction of circulating superoxide dismutase activity in type 2 diabetic patients with microalbuminuria and its modulation by telmisartan therapy," Hypertension Research, vol. 34, no. 12, pp. 13021308, 2011.

[19] M. Nishimura, "Breeding of mice strains for diabetes mellitus," Experimental Animals, vol. 18, pp. 147-157, 1969.

[20] T. Ito, M. Tanimoto, K. Yamada et al., "Glomerular changes in the KK-A ${ }^{y} / \mathrm{Ta}$ mouse: a possible model for human type 2 diabetic nephropathy," Nephrology, vol. 11, no. 1, pp. 29-35, 2006.

[21] M. Tanimoto, T. Gohda, S. Kaneko et al., "Effect of pyridoxamine $(\mathrm{K}-163)$, an inhibitor of advanced glycation end products, on type 2 diabetic nephropathy in KK- $A^{y} / \mathrm{Ta}$ mice," Metabolism, vol. 56, no. 2, pp. 160-167, 2007.

[22] M. Zhang, S. Hagiwara, M. Matsumoto et al., "Effects of eicosapentaenoic acid on the early stage of type 2 diabetic nephropathy in $\mathrm{KKA}^{y} / \mathrm{Ta}$ mice: involvement of antiinflammation and antioxidative stress," Metabolism: Clinical and Experimental, vol. 55, no. 12, pp. 1590-1598, 2006.

[23] S. Hagiwara, T. Gohda, M. Tanimoto et al., "Effects of pyridoxamine (K-163) on glucose intolerance and obesity in high-fat diet C57BL/6J mice," Metabolism, vol. 58, no. 7, pp. 934-945, 2009.

[24] Q. Fan, J. Liao, M. Kobayashi et al., "Candesartan reduced advanced glycation end-products accumulation and diminished nitro-oxidative stress in type 2 diabetic KK/Ta mice," Nephrology Dialysis Transplantation, vol. 19, no. 12, pp. 30123020, 2004.

[25] E. R. Weibel, Ed., Stereological Methods. Practical Methods for Biological Morphometry, vol. 1, Academic Press, London, UK, 1979.

[26] H. Huang, K. T. Iida, H. Sone, T. Yokoo, N. Yamada, and R. Ajisaka, "The effect of exercise training on adiponectin receptor expression in $\mathrm{KKA}^{y}$ obese/diabetic mice," Journal of Endocrinology, vol. 189, no. 3, pp. 643-653, 2006.

[27] P. Boor, P. Celec, M. Behuliak et al., "Regular moderate exercise reduces advanced glycation and ameliorates early diabetic nephropathy in obese Zucker rats," Metabolism, vol. 58, no. 11, pp. 1669-1677, 2009.

[28] C. N. Lumeng, J. L. Bodzin, and A. R. Saltiel, "Obesity induces a phenotypic switch in adipose tissue macrophage 
polarization," Journal of Clinical Investigation, vol. 117, no. 1, pp. 175-184, 2007.

[29] H. S. Park, J. Y. Park, and R. Yu, "Relationship of obesity and visceral adiposity with serum concentrations of CRP, TNF- $\alpha$ and IL-6," Diabetes Research and Clinical Practice, vol. 69, no. 1, pp. 29-35, 2005.

[30] F. Moien-Afshari, S. Ghosh, M. Khazaei, T. J. Kieffer, R. W. Brownsey, and I. Laher, "Exercise restores endothelial function independently of weight loss or hyperglycaemic status in db/db mice," Diabetologia, vol. 51, no. 7, pp. 1327-1337, 2008. 


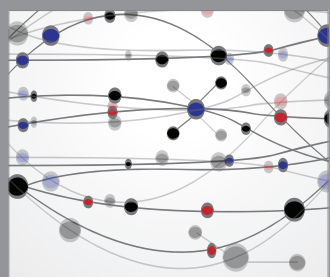

The Scientific World Journal
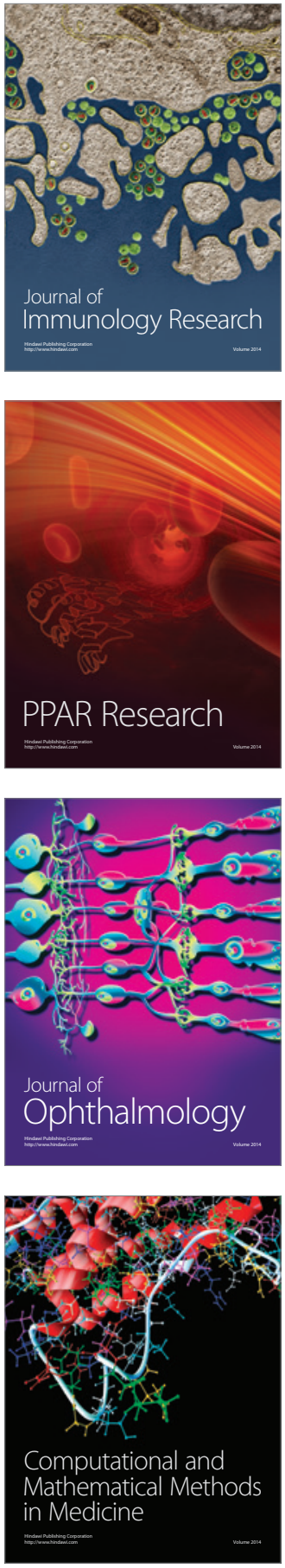

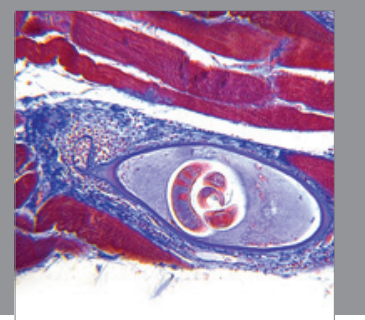

Gastroenterology

Research and Practice
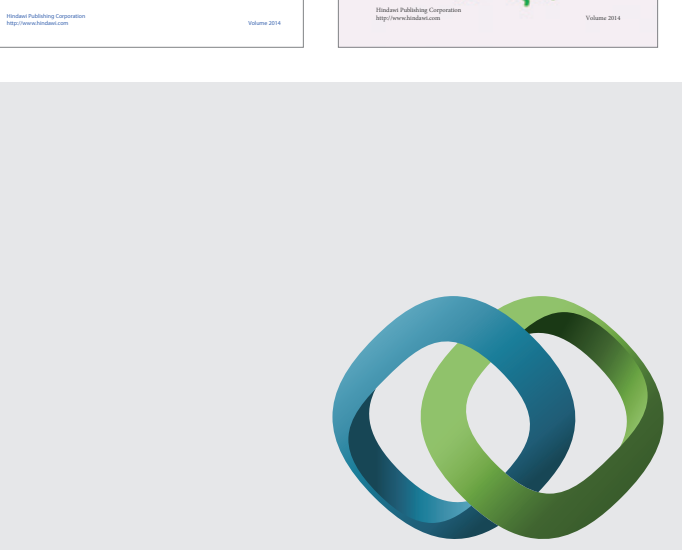

\section{Hindawi}

Submit your manuscripts at

http://www.hindawi.com
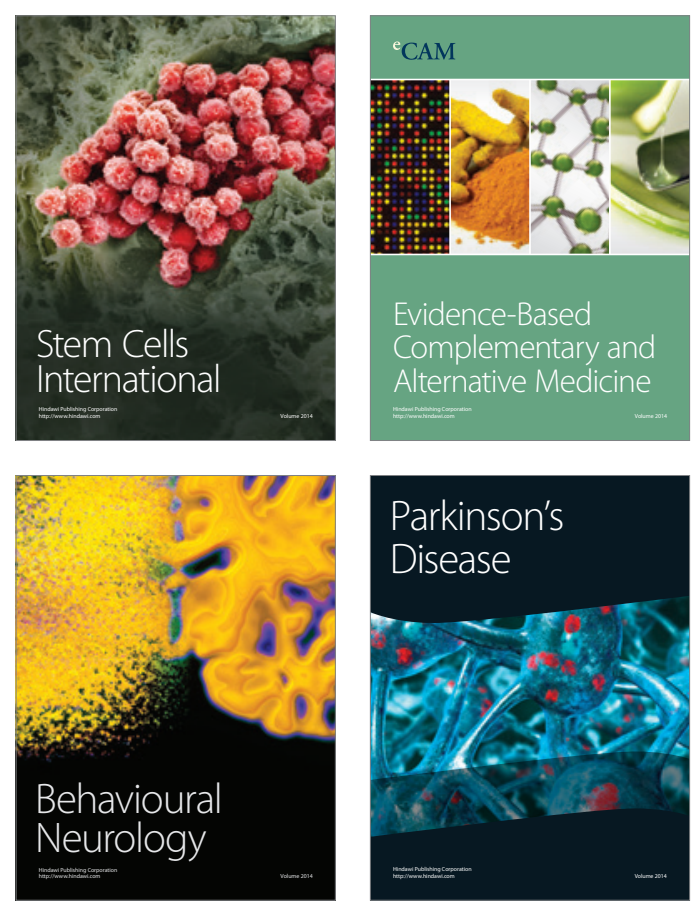

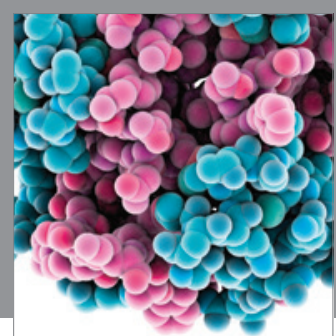

Journal of
Diabetes Research

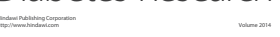

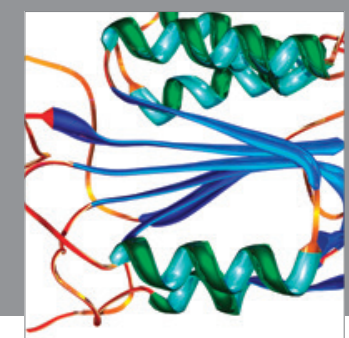

Disease Markers
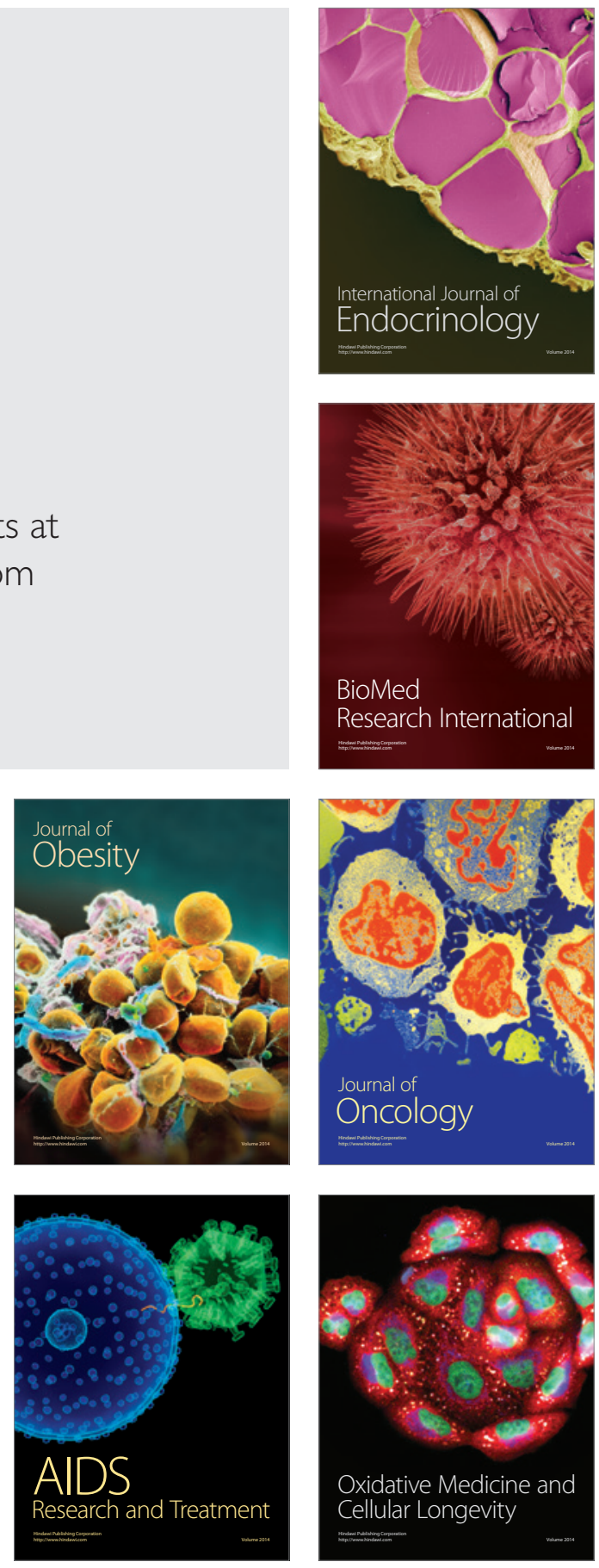